\title{
BMJ Open Activities and programmes that support the emotional wellness and well-being of refugees, immigrants and other newcomers within settlement agencies: a scoping review protocol
}

\author{
Suzanne Goopy, ${ }^{1}$ Cesar Suva, ${ }^{1,2}$ K. Alix Hayden, ${ }^{3}$ Halley Silversides (D) , \\ Katerina Palova ${ }^{2}$
}

To cite: Goopy S, Suva C, Hayden KA, et al. Activities and programmes that support the emotional wellness and wellbeing of refugees, immigrants and other newcomers within settlement agencies: a scoping review protocol. BMJ Open 2020;10:e033377. doi:10.1136/ bmjopen-2019-033377

- Prepublication history for this paper is available online. To view these files, please visit the journal online (http://dx.doi org/10.1136/bmjopen-2019033377).

Received 02 August 2019

Revised 14 July 2020

Accepted 29 July 2020

\section{Check for updates}

(C) Author(s) (or their employer(s)) 2020. Re-use permitted under CC BY-NC. No commercial re-use. See rights and permissions. Published by BMJ.

${ }^{1}$ Faculty of Nursing, University of Calgary, Calgary, Alberta,

Canada

${ }^{2}$ Research and Program Development, The Immigrant Education Society, Calgary,

Alberta, Canada

${ }^{3}$ Libraries and Cultural

Resources, University of Calgary, Calgary, Alberta, Canada

Correspondence to

Dr Suzanne Goopy;

sgoopy@ucalgary.ca

\section{ABSTRACT}

Introduction Obstacles to successful settlement-social isolation, language hardship, issues with employment, housing questions, transportation, barriers to health, education and government service access-all potentially play a role in emerging physical and mental health problems. The objective of this scoping review is to map the available evidence in order to provide an overview of the services and resources offered to refugees, immigrants and other newcomers by settlement agencies to support emotional wellness and well-being.

Methods and analysis The protocol to be followed for this scoping review is based on the Joanna Briggs Institute to provide a map of the current and emergent literature, and examine the extent, range and nature of this literature. The proposed scoping review will also identify the gaps in research pertaining to the emotional wellness of refugees, immigrants and other newcomers as well as summarise and disseminate research findings and provide direction for future reviews. Key databases for this scoping review include APA PsycINFO, Medline, Embase, Cumulative Index of Nursing and Allied Health Literature Plus, Academic Search Complete, and Education Research Complete. The database search start and end dates for this scoping review will be from inception to July 2020. The article searches will take place between August and October 2020. Ethics and dissemination Ethics is not required as the research will not involve human or animal subjects. The research is a scoping review, and thus relies on published and grey literature studies and documents. The findings of this proposed scoping review will be disseminated through future publications as well as presentations to relevant stakeholders, including immigrant serving agencies. We anticipate that this scoping review will identify gaps in research pertaining to the emotional wellness of refugees, immigrants and other newcomers. The results of this review will be the first comprehensive recent survey of emotional wellness practices employed by settlement agencies.

\section{INTRODUCTION}

Research since the early twentieth century has revealed that immigrants can frequently
Strengths and limitations of this study

- Studies will only be reviewed if they are in English and/or French-there is the possibility that some relevant studies may be missed.

- The team consists of content experts from both the academic and non-profit immigrant-serving sector as well as a methodological expert health sciences librarian.

- Following rigorous Joanna Briggs Institute (JBI methodology and using software (Covidence and JBI-SUMARI) for review.

- Comprehensive data collection—searching multiple sources and will include grey literature.

experience mental health issues to a higher degree than local populations. ${ }^{12}$ Although such individuals might arrive in Canada with a lower average incidence of mental health issues compared with locals, these can increase due to the stress, insecurity and precariousness that often accompanies the settlement process. ${ }^{3}$ This is precipitated by the loss of longestablished and diverse social and emotional ties that existed in newcomers' home countries but are absent at the settlement destination. ${ }^{4}$ Rates of mental illness can be especially high among immigrants who are most vulnerable, such as seniors, women and children. There can also be variation in experiences between cultural groups, depending on similarities with the host culture, such as language and religion. ${ }^{4}$ Obstacles to successful settlementsocial isolation, language hardship, issues with employment, housing questions, transportation, barriers to health, education, government service access, and the overall experience of migrating to a new country-all potentially play a role in emerging physical and mental health problems. ${ }^{3}$ Recent immigrants, for example, 
are more likely to suffer disproportionally with the cost of housing, and with its consequent impact on physical and emotional health. ${ }^{5}$

Vulnerable newcomers, such as refugees, undocumented immigrants and asylum seekers, can be reticent to approach mainstream health and social services because of actual and imagined consequences. These imagined consequences can include impacts on their immigration status, ${ }^{6}$ while actual ones may include financial liability. ${ }^{7}$ Difficulties in expressing themselves in English, as well as hesitancy to share highly personal information with health professionals with whom they have no established relationship, can be nearly insurmountable barriers for many newcomers. ${ }^{7}$ While the Canada Health Act recommends that approaches to service delivery include accommodation for cultural and linguistic diversity, there is still a high degree of inconsistency in the provision of these services that ensure access to newcomers of various backgrounds. ${ }^{6}$

Some studies have advocated the fostering of a heterogeneous and diversified series of social connections for a more accommodating, culturally appropriate and comprehensive provision of health and support services for immigrant populations. ${ }^{8}$ The presence of a broad network of 'weak ties', some authors have argued, is, in fact, more viable in ensuring community health access for caregiving women, for example, than of strong ties in a more limited network. ${ }^{9}$

In a similar vein and for a host of other contextual reasons, settlement agencies in Canada have, in the past few years, developed services and programming that focus on the support of newcomer emotional health and wellness. However, these practices are diverse, limited, underdeveloped, often inconsistent and sometimes redundant, which can have a detrimental effect on how newcomers engage with, and ultimately benefit from, these services. A comprehensive examination of these practices over a wide range of contexts can inform researchers as well as practitioners about the most effective ways of ensuring the emotional wellness of the newcomers they serve.

\section{METHODS AND ANALYSIS}

This proposed scoping review will be conducted in the following manner, adhering to the process/guidelines of the Joanna Briggs Institute (JBI) scoping review methodology:

1. Research question identification.

2. Identification of relevant studies.

3. Selection of studies to be included.

4. Extraction of relevant data from included studies.

5. Summary, compilation and reporting of results.

6. Sharing of results with stakeholders.

\section{Review title}

Activities and programmes that support the emotional wellness of refugees, immigrants and other newcomers within settlement agencies: A scoping review protocol

\section{Review objective}

The aim of this proposed scoping review is to provide a map of the existing literature, and examine the extent, range and nature of the current and emergent literature. This scoping review will also identify the gaps in research pertaining to the emotional wellness and well-being of refugees, immigrants and other newcomers as well as summarise and disseminate research findings. It will also provide the basis for a summary of the most successful practices and initiatives employed to support newcomer emotional wellness available in the literature, as well as the parameters of their success. The discussion of these parameters will also identify barriers as well as facilitators to the success of this programming. While the scoping review will be primarily used to inform knowledge users by identifying gaps in the research knowledge base, ${ }^{10}$ clarifying key concepts ${ }^{11}$ and reporting on the types of evidence that address and inform the delivery of integration services across a range of disciplinary and sub-disciplinary areas, ${ }^{12}$ it may also be used to provide direction for future systematic reviews.

\section{Review question}

JBI suggests using the mnemonic "population, concept and context' to inform the research question as well as the inclusion criteria when conducting a scoping review. ${ }^{13}$ For our review, the population is refugees, immigrants and other newcomers, the concept is emotional wellness and emotional well-being, and the context is settlement agencies. Therefore, our research question is as follows:

Which practices aimed at addressing the emotional wellness and well-being of refugees, immigrants and other newcomers are, or have been, offered by settlement agencies?

\section{Inclusion criteria}

Population

Newcomers, refugees, immigrants who have reached their final destination and who are involved with 'formal' settlement agencies' services will be the focus of the review. Undocumented and illegal immigrants served by more 'informal' community associations and organisations will also be included. This review will focus both on practices at 'formal' organisations as well as 'informal' organisations. 'Formal' organisations are typically not-for-profit settlement agencies that employ permanent staff and receive funding from various levels of government and other nongovernmental funding agencies. 'Informal' organisations are driven primarily by volunteer staff and participants, regardless of their source of funding. While more 'formal' organisations typically have a higher degree of consistency and sustainability within their programmes, volunteer-based community organisations can have varied programming due to their precarious funding and resources. This review will look at both types of organisations, which both hold significant value and contributions to newcomer communities, often in different ways. The participant population includes individuals over 18 or families. Children, without 
a family unit or where services are offered within schools only, will be excluded.

\section{Concept}

Our review will investigate the emotional wellness and well-being of newcomers settling in their final destination country. Studies, programme descriptions or interventions that focus on enhancing well-being, or addressing well-being or barriers/gaps to well-being, will be included. Studies that involve conditions addressed and intervened on by formal health service professionals such as those concerning psychiatric mental disorders will be excluded. Further, studies that focus on interventions for diagnosable conditions, communicable diseases or other diseases and physical illnesses will be excluded. Studies that consider mental state but without Diagnostic and Statistical Manual-5 criteria will be included. Studies focused on participants' thinking around ethnicity, identity, identification and ethnic identification will be excluded in the scoping review.

\section{Context}

The review focuses on newcomers in formal settlement agency contexts within their final destination country. As we are looking at the emotional wellness and well-being of refugees, immigrants and other newcomers, we will include studies that deal with families (adults and their children) or adults within the context of formal settlement agencies and services provided by settlement agencies within the destination country. Community-based programmes or research will be included because of the study's interest in non-governmental assistance within Canadian communities aimed at the successful integration of newcomers. As the study examines service delivery, studies can be about an intervention or formalised programme. School-based programmes for children will be excluded. Services that assist clients in more transitory situations, without consideration for the long-term adjustment and integration of the client, will be excluded. Because of the study's focus on service providers who aim to settle and integrate clients permanently into the local community, studies that consider participants whose place of settlement are refugee camps will not be included.

\section{Types of studies}

All study designs, such as those that are descriptive, qualitative, quantitative, case studies, surveys or using mixed methods, will be included in the review. Further, we will include grey literature, including dissertations, government reports and settlement agency documents or programme descriptions. Systematic reviews and other knowledge synthesis research will be excluded, but the reference lists will be scanned for potentially relevant studies. Studies where participants settle in Europe, North America or Australasia-regions that have comparable forms of governance, degree of economic development and cultural context to Canada-will be included. Studies where participants settle outside of these regions will be excluded. The search strategy will be limited to works published in either
French or English. This review will consider studies that are in either English or French because the study is aimed at use in Canada for both English and French settlement services. There will be no date restrictions.

\section{Search strategy}

The search strategy for data collection for the scoping review will be purposefully comprehensive and exhaustive. As recommended by JBI, our review follows a three-step search strategy. ${ }^{13}$ The first step involved exploring the literature. The team met to discuss and brainstorm potential keywords for the three main concepts: newcomers, wellness and settlement agencies. The team also identified seed articles. An initial search was conducted by an expert health sciences librarian in APA PsycINFO and Google Scholar with the identified keywords. The seed articles were also analysed for keywords and subject headings. Second, the identified keywords and subject headings were then developed into a comprehensive search strategy, with continuous exploring of newly identified keywords through scanning the retrieved results. This was an iterative process, where new keywords and subject headings were added as discovered. This search strategy, in APA PsycINFO, was shared with the team, who provided feedback, and further revisions were undertaken. The APA PsycINFO search was also pilot tested to be sure that it captured the known seed articles. The provisional search strategy is available in table 1 . The APA PsycINFO search strategy will be adapted for each database, with keywords remaining constant across databases, and subject headings being responsive to the controlled vocabulary of the database. The final step will involve snowball searching. Specifically, both reference lists and works cited in included studies will be screened. Citations, references and bibliographies of included studies will also be screened. It is the intent of the reviewers to contact the authors of primary studies or reviews for further information as appropriate.

Preliminary simple searches, focusing on the main concepts (newcomers/immigrants/refugees, wellness/ well-being, settlement) were conducted in numerous databases to identify those which would be most informative. Based on these preliminary searches, the search will be conducted in the following Ovid databases: APA PsycINFO, Medline and Embase. EbscoHost databases to be searched include the following: Cumulative Index of Nursing and Allied Health Literature Plus, Academic Search Complete, Anthropology Plus, Education Research Complete, ERIC, Family \& Society Studies Worldwide, Family Studies Abstracts and SocINDEX. Sociological abstracts will be searched on the ProQuest platform. Scopus will also be searched. Table 1 provides the provisional search strategy for APA PsycINFO.

All search results from the databases will be uploaded into Covidence where it will be automatically deduplicated.

\section{Study selection}

The retrieved studies will be screened in two phases, both of which will use Covidence to assist in the screening process. 


\begin{tabular}{|c|c|c|}
\hline \# & Searches & Results \\
\hline 1 & exp Immigration/ & 20977 \\
\hline 2 & exp MIGRANT FARM WORKERS/ & 423 \\
\hline 3 & exp refugees/ & 5409 \\
\hline 4 & exp Asylum Seeking/ & 464 \\
\hline 5 & (immigrant* or immigration).tw,id. & 30349 \\
\hline 6 & $\begin{array}{l}\text { (refugee }{ }^{\star} \text { or foreign born* or asylum } \\
\text { seek }^{\star} \text { or migrant* or illegal alien*).tw,id. }\end{array}$ & 18807 \\
\hline 7 & $\begin{array}{l}\left.\text { (displaced adj2 (people* or person }{ }^{\star}\right) \text { ). } \\
\text { tw,id. }\end{array}$ & 595 \\
\hline 8 & $\begin{array}{l}\text { (newcomer }{ }^{\star} \text { or economic migrant* or } \\
\left.\text { economic immigrant }{ }^{\star}\right) . t w \text {,id. }\end{array}$ & 2462 \\
\hline 9 & or/1-8 & 47298 \\
\hline 10 & $\begin{array}{l}\text { exp Well Being/ or exp BELONGING/ or } \\
\text { exp Social Integration/ }\end{array}$ & 47053 \\
\hline 11 & $\begin{array}{l}\text { exp HAPPINESS/ or exp Social Capital/ } \\
\text { or exp Sociocultural Factors/ }\end{array}$ & 125310 \\
\hline 12 & $\begin{array}{l}\text { exp LIFE SATISFACTION/ or exp } \\
\text { "Quality of Life"/ or exp Social Support/ }\end{array}$ & 81217 \\
\hline 13 & $\begin{array}{l}\text { exp COPING BEHAVIOR/ or exp Mental } \\
\text { Health/ }\end{array}$ & 106607 \\
\hline 14 & $\begin{array}{l}\text { (social inclusion or well being or } \\
\text { wellbeing or belonging }{ }^{\star} \text { ).tw,id. }\end{array}$ & 104158 \\
\hline 15 & $\begin{array}{l}\text { (acculturation or resettlement or } \\
\text { settlement).tw,id. }\end{array}$ & 15866 \\
\hline 16 & (satisfaction or happiness).tw,id. & 116585 \\
\hline 17 & $\begin{array}{l}\text { (social capital or integration or cultural } \\
\text { capital or cultural code*).tw,id. }\end{array}$ & 95530 \\
\hline 18 & $\begin{array}{l}\text { ("quality of life" or mental health or } \\
\text { wellness or social support or coping). } \\
\text { tw,id. }\end{array}$ & 337843 \\
\hline 19 & or/10-18 & 699901 \\
\hline 20 & $\begin{array}{l}\text { exp DISSATISFACTION/ or exp } \\
\text { SADNESS/ }\end{array}$ & 3966 \\
\hline 21 & $\begin{array}{l}\text { exp Loneliness/ or exp SOCIAL } \\
\text { ISOLATION/ }\end{array}$ & 11153 \\
\hline 22 & $\begin{array}{l}\text { exp SOCIAL STRESS/ or exp } \\
\text { DISTRESS/ }\end{array}$ & 22893 \\
\hline 23 & $\begin{array}{l}\text { exp Anxiety/ or exp FEAR/ or } \\
\text { exp Disadvantaged/ or exp } \\
\text { DISAPPOINTMENT/ }\end{array}$ & 90620 \\
\hline 24 & $\begin{array}{l}\text { (dissatisfaction or dis-satisfaction or } \\
\text { sadness).tw,id. }\end{array}$ & 21615 \\
\hline 25 & $\begin{array}{l}\text { (social adj3 (exclusion or isolation)). } \\
\text { tw,id. }\end{array}$ & 10760 \\
\hline 26 & (loneliness or lonely).tw,id. & 10136 \\
\hline 27 & $\left(\right.$ distress $^{\star}$ or stress $\left.{ }^{\star}\right) . t w, i d$ & 303290 \\
\hline 28 & $\begin{array}{l}\text { (anxious* or worrying* or fear* or afraid } \\
\text { or frightened or nervous }{ }^{\star} \text { ).tw,id. }\end{array}$ & 171714 \\
\hline 29 & $\begin{array}{l}\text { (unsettled or disillusion* or disappoint*). } \\
\text { tw,id. }\end{array}$ & 8995 \\
\hline
\end{tabular}

Continued

\begin{tabular}{|c|c|c|}
\hline \# & Searches & Results \\
\hline 30 & or/20-29 & 542376 \\
\hline 31 & 19 or 30 & 1097619 \\
\hline 32 & (service adj2 provider ).tw,id. & 10490 \\
\hline 33 & $\begin{array}{l}\text { ((English or French) adj2 (teacher }{ }^{\star} \text { or } \\
\text { instructor }{ }^{\star} \text { or provider*)).tw,id. }\end{array}$ & 1815 \\
\hline 34 & $\begin{array}{l}((E S L \text { or FSL) adj2 (teacher* or } \\
\text { instructor }{ }^{\star} \text { or provider*)).tw,id. }\end{array}$ & 315 \\
\hline 35 & $\begin{array}{l}\text { (language adj2 (instruct* or learning or } \\
\text { education*)).tw,id. }\end{array}$ & 15510 \\
\hline 36 & $\begin{array}{l}\text { (translator* or advocat* or helper* or } \\
\left.\text { volunteer }{ }^{\star}\right) . t w, \text { id. }\end{array}$ & 72093 \\
\hline 37 & $\begin{array}{l}\text { ((settlement or resettlement or voluntary } \\
\text { or community) adj2 worker }) \text {.tw,id. }\end{array}$ & 1783 \\
\hline 38 & $\begin{array}{l}\left(\left(\text { refugee }^{\star} \text { or immigrant }^{\star} \text { or settlement }\right.\right. \\
\text { or resettlement } \\
\left.\text { worker }^{\star} \text { or adj2 }\left(\text { agent }^{\star} \text { or service }{ }^{\star}\right)\right) \text { or } \text {.tw,id. }\end{array}$ & 969 \\
\hline 39 & community member*.tw,id. & 5609 \\
\hline 40 & (not-for-profit or nonprofit).tw,id. & 5255 \\
\hline 41 & (child mind* or babysit*).tw,id. & 331 \\
\hline 42 & $\begin{array}{l}\left(\text { child adj2 (minder }{ }^{*} \text { or minding or }\right. \\
\left.\text { worker }^{\star}\right) \text { ).tw,id. }\end{array}$ & 1811 \\
\hline 43 & babysit*.tw,id. & 224 \\
\hline 44 & exp NONPROFIT ORGANIZATIONS/ & 2264 \\
\hline 45 & exp VOLUNTEERS/ & 4519 \\
\hline 46 & exp Community Services/ & 31548 \\
\hline 47 & or/32-46 & 141652 \\
\hline 48 & 9 and 31 and 47 & 2155 \\
\hline 49 & limit 48 to (english or french) & 2060 \\
\hline
\end{tabular}

Database(s): PsycINFO 1806 to May Week 32019.

In the first phase, two content experts (CS and HS) will independently screen these studies by title and abstract, applying the inclusion/exclusion criteria to each record. Prior to commencing screening, the content experts will pilot screen a sample of 100 records, applying the inclusion/exclusion criteria. The content experts will discuss their decisions, and revise or further define inclusion/ exclusion criteria if required. Title and abstract screening may commence when an inter-rater agreement of $85 \%$ or greater has been established. Discrepancies in screening will be decided through consensus, or a third content expert (SG) will be consulted for discussion. Records that meet the inclusion criteria, or were inconclusive, will move to the second phase of screening, which involves full-text screening. Again, two content experts (KP and SG) will independently apply the inclusion/exclusion criteria to the full-text of each study. Disagreements will be determined through consensus, or discussion with a third content expert (HS or CS). Search results, screening results for both title/abstract and full-text screening will be reported 


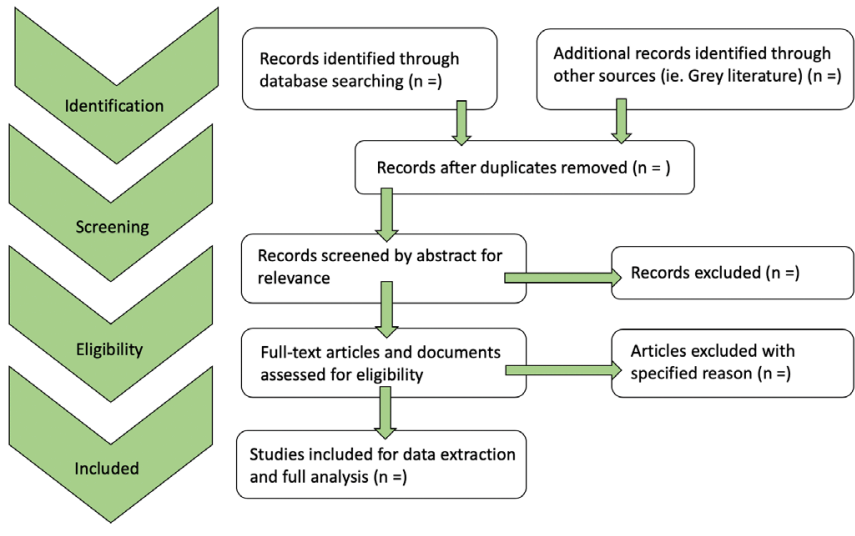

Figure 1 Preferred Reporting Items for Systematic Review and Meta-Analysis Protocols flow diagram demonstrating the planned screening-to-extraction activities of the scoping review.

in the Preferred Reporting Items for Systematic Review and Meta-Analysis Protocols (PRISMA) flow diagram as demonstrated in figure $1 .^{14}$

After screening has been completed, reviewers will extract the data from each study using JBI-SUMARI to create a consistent framework. This framework may be further refined at the reviewing stage and modified accordingly. Box 1 outlines the categories in which reviewers will extract data.

The methodology and results of the scoping review will be presented in accordance with the reporting guidelines outlined in the PRISMA Extension for Scoping Reviews checklist. ${ }^{15}$ Data will be analysed using narrative synthesis, guided by Popay et $a l \mathrm{~s}^{16}$ approach. These researchers note that narrative synthesis is 'an approach to the systematic review and synthesis of findings from multiple studies that relies primarily on the use of words and text to summarise and explain the findings of the synthesis'.

\section{Patient and public involvement}

This proposed scoping review protocol was written without public involvement. The public was not asked to comment on the design of the scoping review, nor were they invited to contribute to the reading or writing of this document.

\section{Box 1 Data extraction categories}

Author(s).

- Year of publication.

- Origin/Country of Origin (where the study was published or conducted).

- Aims/purpose.

- Study population and sample size (if applicable).

- Methodology/methods.

- Intervention type, comparator and details of these (eg, type of programming or service) (if applicable).

- Duration of the intervention (if applicable).

- Outcomes and details of these (eg, how measures) (if applicable).

Key findings that relate to the scoping review question/s.

\section{Ethics and dissemination}

As this is a proposed scoping review and does not involve human or animal subjects, an ethics board approval was not necessary and thus not obtained. The findings of this proposed scoping review will be disseminated through a future publication as a completed scoping review. Findings will also be communicated to immigrant-serving agencies and those with interest in immigration issues at future conferences. The results of this review will be the first comprehensive recent survey of emotional wellness practices employed by settlement agencies. This scoping review will also be used to directly inform future research on newcomer emotional wellness and well-being.

\section{DISCUSSION}

A wide scope of practices is employed by settlement agencies to address the emotional wellness of newly arrived immigrants and refugees. This proposed scoping review will generate findings that will aid in identifying settlement agencies' activities and programmes that support agencies who undertake work with the emotional wellness of refugees, immigrants and other newcomers as described in academic and grey literature.

While the area of mental health within this population has received a significant amount of research, little research attention has been paid to emotional wellness. This proposed scoping review, therefore, focuses on 'emotional wellness' practices as opposed to those aimed at newcomer 'mental health'. The reason for this is that often settlement agency staff do not have the professional resources to address clinical mental health issues that can often be present in their client population, particularly in those clients who come from conflict zones or have been forced to migrate to their new country. There is much established research, however, that suggests the value of preventative measures in mental health, ${ }^{17}{ }^{18}$ including when addressing social isolation and cultural anxiety. It is often within this area that settlement agencies act, an examination of which can provide valuable insight into the integration process of many new immigrants.

The results of this scoping review will offer comprehensive analysis of emotional wellness practices employed by settlement agencies. The findings generated will also be useful to policymakers, academics, researchers and programme implementers who work with refugees, immigrants and other newcomers within resettlement organisations or in other similar capacities. These can provide a picture of which approaches used by agencies are more common and sustainable in particular contexts. It will also reveal which contexts agencies who operate emotional wellness supports in are more common. The ways in which funder reporting impacts service delivery and programme design, for example, can reveal sector-wide patterns and practices that might be similar. These mirrored structures, in turn, can lead to specific outcomes in clients. Evidence generated through this review will offer a critical foundation from which primary prevention strategies, which directly 
impact the social and ecological determinants of health and emotional wellness, may be built.

For policymakers, practitioners, agencies and other stakeholders, this proposed scoping review can also help identify gaps in practices where needs remain inadequately addressed or examined. A comprehensive overview of practices examined in depth in the literature can serve as a point of reference for agencies who may only have their local experiences and contexts to draw from in determining interventions on emotional wellness and well-being issues. Agencies may also refer to studies in the review to find future effects and impacts on interventions they may be considering in order to make more informed choices in terms of approach. It is acknowledged by the authors that this audience does not typically access academic literature. As such study results will be disseminated in briefings and presentations at industry-oriented (as opposed to primarily academic) conferences. The involvement of the two authors of this review whose affiliation lies with a settlement agency facilitates access to this audience of stakeholders.

\section{STRENGTHS AND LIMITATIONS}

Results from this scoping review can identify and assess the wide scope of practices employed by settlement agencies to address the emotional wellness of new immigrants and refugees. These practices and programming may, however, not be documented completely and extensively in published academic literature, but rather are available in grey literature that includes government reports and project results summaries held at the agency, funder or community level. As access to these documents may be challenging, the researchers acknowledge that this may be a limitation to the study.

A synthesis and analysis of these practices and approaches by the authors will also provide a comprehensive summary of the most successful of these practices as they are found in the established academic literature. However, this study will only include articles and reports published in English and French, and therefore works in other languages will not be reviewed. Additional strengths and limitations will be identified during the conduct of the scoping review itself.

Acknowledgements The authors would like to acknowledge The Immigrant Education Society. The authors also received grants from other funding bodies, including municipal and provincial governments of Canada, in addition to private foundations.

Contributors SG conceptualised the review and was involved in drafting and editing the protocol paper, guided the development and refinement of the research question, revised the title, guided the development of inclusion/ exclusion criteria, the establishment of the review objectives, final preparation of protocol for submission. KAH provided methodological guidance throughout the development of the protocol, wrote the data collection component and developed the search strategy. HS was involved in drafting and editing the protocol paper, the development of inclusion/exclusion criteria, strengths and limitations, PRISMA chart, final edits and reference clean-ups and proofreading. CS and KP were involved in drafting and editing the protocol, reviewing of the background literature and giving contextualisation to the study within the third sector, and involved in the development of inclusion and exclusion criteria.

Funding This work was supported by Immigration, Refugees and Citizenship Canada (IRCC) grant number X194857003. The co-authors from The Immigrant Education Society (formerly the Calgary Immigrant Educational Society) are fulltime employees for this agency and IRCC is a major funder for their organisation.

Patient consent for publication Not required.

Provenance and peer review Not commissioned; externally peer reviewed.

Open access This is an open access article distributed in accordance with the Creative Commons Attribution Non Commercial (CC BY-NC 4.0) license, which permits others to distribute, remix, adapt, build upon this work non-commercially, and license their derivative works on different terms, provided the original work is properly cited, appropriate credit is given, any changes made indicated, and the use is non-commercial. See: http://creativecommons.org/licenses/by-nc/4.0/.

ORCID iD

Halley Silversides http://orcid.org/0000-0002-6846-8078

\section{REFERENCES}

1 Odegaard O. Emigration and insanity: a study of mental disorders among Norwegian-born population in Minnesota. Copenhagen: Levin \& Munksgaard, 1932.

2 Finlayson J. Is migration good for you? A psychiatric and historical perspective. In: Harper M, ed. Migration and mental health: past and present. London, UK: Palgrave MacMillan, 2016: 239-58.

3 Hordyk SR, Hanley J, Richard Éric. "Nature is there; its free": Urban greenspace and the social determinants of health of immigrant families. Health Place 2015;34:74-82.

4 Bhugra D, Wojcik W, Gupta S, et al. culture shock and culture conflict: Adjustments and reactions. In: Bhugra D, Gupta, S D, eds. Migration and mental health. Cambridge: Cambridge University Press, 2011: 139-48.

5 Bryant T. Housing and health. In: Raphael D, ed. Social determinants of health: Canadian perspectives. Toronto: Canadian Scholars Press, 2016: 360-87.

6 Oxman-Martinez J, Hanley J, Lach L, et al. Intersection of Canadian policy parameters affecting women with precarious immigration status: a baseline for understanding barriers to health. J Immigr Health 2005;7:247-58.

7 Neufeld A, Harrison MJ, Stewart MJ, et al. Immigrant women: making connections to community resources for support in family caregiving. Qual Health Res 2002;12:751-68.

8 Lynam MJ. Support networks developed by immigrant women. Soc Sci Med 1985;21:327-33.

9 Granovetter M. The strength of weak ties: a network theory revisited. Sociological Theory 1983;1:201-33.

10 Crilly RG, Hillier LM, Mason M, et al. Prevention of hip fractures in long-term care: relevance of community-derived data. J Am Geriatr Soc 2010;58:738-45.

11 de Chavez AC, Backett-Milburn K, Parry O, et al. Understanding and researching wellbeing: its usage in different disciplines and potential for health research and health promotion. Health Educ J 2005;64:70-87

12 Decaria JE, Sharp C, Petrella RJ. Scoping review report: obesity in older adults. Int $J$ Obes 2012;36:1141-50.

13 Joanna Briggs Institute. The Joanna Briggs Institute reviewers' manual: Methodology for JBI scoping reviews, 2015. Available: https://libguides.library.kent.edu/ld.php?content_id=40149082 [Accessed Jul 2019].

14 Moher D, Liberati A, Tetzlaff J, et al. Preferred reporting items for systematic reviews and meta-analyses: the PRISMA statement. PLoS Med 2009;6:e1000097.

15 Tricco AC, Lillie E, Zarin W, et al. PRISMA extension for scoping reviews (PRISMA-ScR): checklist and explanation. Ann Intern Med 2018;169:467-73.

16 Popay J, Roberts H, Sowden A, et al. Guidance on the conduct of narrative synthesis in systematic reviews: a product from the ESRC methods programme. Lancaster: Institute for Health Research, Lancaster University, 2006.

17 Citrome L, Yeomans D. Do guidelines for severe mental illness promote physical health and well-being? J Psychopharmacol 2005;19:102-9.

18 Weine SM. Developing preventive mental health interventions for refugee families in resettlement. Fam Process 2011;50:410-30. 